\title{
Size Effect in Plasticity of Gold at the Sub-Micron Scale
}

Julia R. Greer', Arief Budiman², and Professor William D. Nix².

\author{
Palo Alto Research Center (PARC) \\ 3333 Coyote Hill Road, Palo Alto, CA 94304 \\ ${ }^{2}$ Department of Materials Science and Engineering \\ Stanford University, Stanford, CA 94305
}

The results of both experimental studies and molecular dynamics simulations indicate that crystals exhibit strong size effects at the sub-micron scale. Size effects in indentation, torsion and bending have been understood in terms of the non-uniformity of the deformation, which sets up strain gradients leading to hardening [1]. Size effects are also found in thin films, where the strength scales inversely with film thickness and is usually attributed to the confinement of dislocations by the substrate [2]. Size effects are observed for pristine crystals, as well [3]. In the earliest stages of nanoindentation, for example, the crystal volume is extremely small and can be dislocation-free, requiring very large stresses to nucleate new dislocations. In addition, classic experiments on the initially dislocation-free metal whiskers indicated that whiskers with smaller diameters yielded at higher stresses [4]. Finally, several molecular dynamics simulations [5] and more recent experiments on small pillars [6,7] support the tenet that smaller is stronger. In spite of much progress on size effects research there is still no unified theory for plastic deformation at the sub-micron scale.

In this work, we focus on size effects arising in unconstrained geometries, in the absence of strain gradients, and with non-zero initial dislocation densities. Gold nanopillars ranging in diameter between $200 \mathrm{~nm}$ and several micrometers were fabricated from bulk gold and epitaxial gold films on $\mathrm{MgO}$ substrates using focused ion beam (FIB) machining. Some of the representative gold pillars are shown in Fig. 1.
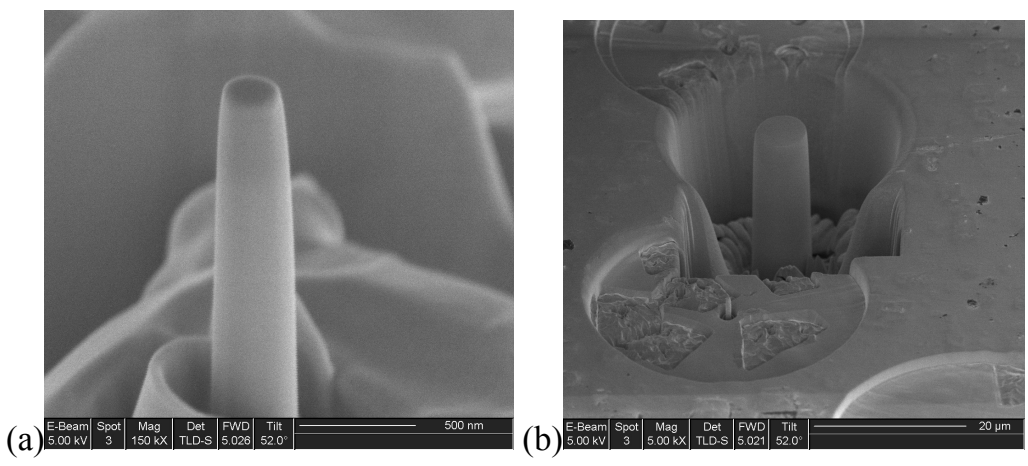

FIG. 1. (a) A representative $<001>$-oriented gold pillar machined in the FIB. Pillar diameter $=290$ $\mathrm{nm}$, pillar height $=1.2$ m. (b) A large pillar (7.45 m diameter) and a small pillar $(250 \mathrm{~nm}$ diameter).

These pillars are subsequently compressed using a Nanoindenter fitted with a custom-fabricated diamond flat punch. The load-displacement data obtained during the compression experiments are then converted to uniaxial stresses and strains using the assumption that the plastic volume is conserved throughout this mostly-homogeneous deformation. These small pillars are found to plastically deform in uniaxial compression at stresses as high as $800 \mathrm{MPa}$, a value $\sim 50$ times higher than for bulk gold. Stress- 
strain curves of FIB pillars whose diameters range between $290 \mathrm{~nm}$ and $7450 \mathrm{~nm}$ as well as the strength of bulk gold at $2 \%$ strain are presented in Fig. 2 (a).

(a)
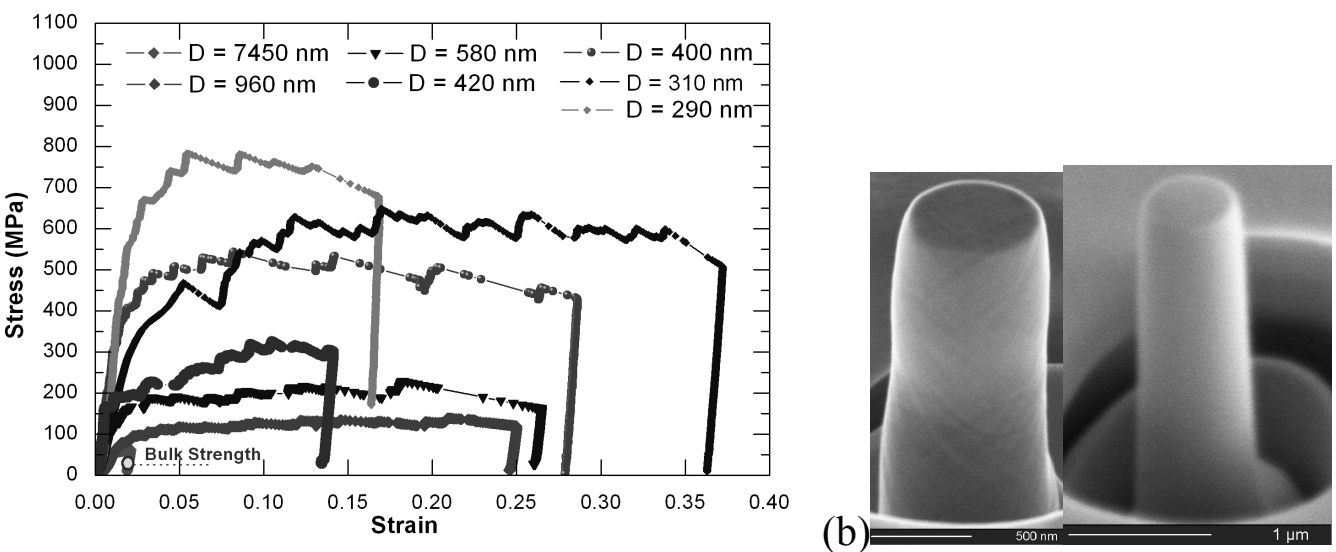

FIG. 2 (a) Stress-strain behaviour of <001>-oriented pillars: flow stresses increase significantly as the pillar diameter is reduced. (b) SEM image of the same pillar before and after deformation. Slip lines in multiple orientations are clearly present in the deformed states and indicate a homogeneous shape change.

We believe that these high strengths are controlled by the process of hardening by dislocation starvation. In this mechanism, once the sample is small enough, the mobile dislocations have a higher probability of annihilating at a nearby free surface than of multiplying and being pinned by other dislocations. When the starvation conditions are met, plasticity is accommodated by the nucleation and motion of new dislocations rather than by motion and interactions of existing dislocations, as is the case for bulk crystals. Cross-sectional TEM studies of the deformed pillars are found to be void of any mobile dislocations and show only two dislocations whose Burgers vectors are perpendicular to the loading axis and thus not driven by the applied stress. The degree of homogeneity of pillar deformation was studied via $\mathrm{X}$-ray synchrotron radiation. This paper will discuss the proposed dislocation starvation hypothesis as a possible plasticity mechanism at the sub-micron and below scales.

1. See, for example, N.A. Stelmashenko, M.G. Walls, L.M. Brown, Y.V. Millman, Acta Mat. 41, 2855 (1993); Y. Huang, Z. Xue, H. Gao, W.D. Nix, Z.C. Xia, J. Mater. Res. 15, 1786 (2000).

2. See, for example, W.D. Nix, Metall. Trans. A 20A, 2217 (1989) and Y. Yu, F. Spaepen, J. Appl. Phys. 95, 2991 (2003).

3. E. Arzt, G. Dehm, P. Gumbsch, O. Kraft, D. Weiss, Progress in Mater. Sci. 46, 283 (2001).

4. S.S. Brenner, J. Appl. Phys. 27, 1484 (1956); S.S. Brenner, R. Doremus, B.W.

Roberts, D.Turnbull, Growth and Perfection of Crystals (John Wiley, New York, 1958), p.157.

5. V.Deschpande, A. Needleman, E. Van der Giessen, E., J. Mech. Phys. Solids, (to be published)

6. M.D. Uchic, D.M. Dimiduk, J.N. Florando, W.D. Nix, Science 305, 986 (2004).

7. J.R. Greer, W.C. Oliver, W.D. Nix, W.D., Acta Mat. 53, 1821 (2005). 